\title{
CÓMO MEDIR LA RENTABILIDAD DE LA INVERSIÓN EN EDUCACIÓN: UNA NOTA ACADÉMICA BASADA EN EL VAN Y LA TIR
}

\author{
Nicko A. Gomero Gonzales \\ Docente-Facultad de Ciencias Contables
}

\begin{abstract}
RESUMEN
En el siguiente artículo se plantea un modelo para medir la rentabilidad de la inversión en educación, se hace un enfoque netamente teórico de tipo macroeconómico para pasar a desarrollar y explicar esta rentabilidad en términos del Valor Actual Neto y de la Tasa Interna de Rentabilidad, ya que son los mejores indicadores para tomar una decisión de inversión. El modelo que se plantea es netamente académico, pero no deja de tener el valor agregado suficiente para aplicarlo a la vida real cuando se quiera realizar emprendimentos referidos al campo de la capacitación. Este enfoque es de utilidad para aquellos que están involucrados en el tema de las inversiones o, en general, para los que quieren tener a la mano una herramienta de gran operatividad para tomar decisiones de esta naturaleza.
\end{abstract}

Palabras clave: Nivel educativo y crecimiento económico, educación e inversión, capital humano, rentabilidad de la inversión educativa.

\section{INTRODUCCIÓN}

La promoción de la educación, la capacitación y la investigación, ha sido un renglón constante en la política de desarrollo económico durante varias décadas de muchos gobiernos. Su nivel de importancia ha sido reconocido como crucial por los economistas desde los tiempos de Adam Smith. Sin embargo, es solo recientemente, durante los años sesenta, principalmente con el trabajo de Gary Becker, Premio Nobel en 1992, que comienza un tratamiento sistemático y riguroso del problema

El trabajo de Becker introduce la idea de capital humano. Se postula que los individuos acumulan durante su vida un cierto nivel de acervo. Este se encuentra conformado por susconocimientos, experienciasy habilidades.
Los individuos consiguen este capital procesando sus experiencias cotidianas, como resultado de su trabajo $y$, especialmente, con la ayuda de mecanismos y sistemas formales de educación.

Esta acumulación de capital humano tiene dos consecuencias importantes. Una de ellas es que determina la productividad de los trabajadores. Resulta obvio que hay una relación directa entre capital humano y productividad. La segunda, desde la perspectiva de una mayor agregación, es decir, desde un punto de vista macroeconómico, como decimos los economistas, se encuentra en relación a la cantidad de bienes y servicios que una economía puede producir; y esta depende, entreotras cosas, del capital humano, cuyoinsumoprincipal es una educacióndecalidad. 
Este enfoque teórico ha permitido explicar dos regularidades estadísticas sumamente importantes. La primera de estas regularidades se refiere a la existencia de una relación positiva entre salariosynizeleducatizo. En promedio, personas con un mayor nird educativo tienen también un mayor salario. Estudios empíricos muestran este resultado en prácticamente todas las economías del mundo. Naturalmente, este es un resultado promedio que no es necesariamente válido para todos los individuos. Frecuentemente, los educadonesson un claro contraegemplode individuos ayo nivel educativo no se corresponde con su nizd salarial.

La otra notable regularidad estadística es que hay una relación positiva entre el nivel educativo y el crecimiento económico. Estudios empíricos realizados sobre un gran número de países muestran una relación positiva entre nivel educativo, crecimiento económico y riqueza. Los países ricos tienen también un alto nivel educativo. Más aún, los resultados empíricos no sugieren que estos países tienen un alto nivel educativo porque son ricos. Por el contrario, estos resultados sugieren que estos países son ricos porque tienen un alto nivel educativo.

Estos resultados han sido firmemente incorporados en las teorías del crecimiento económico. La conclusión es ineludible: el crecimiento y el bienestar de los individuos y de las sociedades no son posibles sin alcanzar un considerable nivel educativo. Esta valoración de la Educación como una inversión para el futuro crecimiento y bienestar es tan fuerte en los economistas que muchos han señalado que los gastos del Estado en educación no debieran ser registrados comogastos corrientes, sino que como inversión; al igual que los gastos en construcción de caminos, puentes, aeropuertos y otras infraestructuras físicas de largo plazo.
Sin duda, la educación, en términos macroeconómicos, es fundamental para dar sustento al crecimiento y desarrollo económico, pero también es importanteque el individuo conozca su retorno o rendimiento por el esfuerzo que realiza, porque al final de cuentas en forma individual o microeconómica emprende una inversión. y como taldebede tener un retorno favorable para tomar una decisión en este mismo sentido. En el presente artículo se abordará este tema, dando mayor énfasis al Valor Actual Neto y a la Tasa Interna de Retorno. ya que son de los instrumentos más importantes para medir la rentabilidad de una inversión. Definitivamente la educación o capacitación es una inversión, ya sea a nivel macro como a nivel micro.

ESTIMACIÓN DELA RENTABILIDAD DE LA EDUCACIÓN BASADA EN LA TASA INTERNA DE RETORNO (TIR): UN ENFOQUE GENERAL

El efecto de la educación sobre el desarrollo económico tradicionalmente ha sido medido a través del método de la Tasa Interna de Retorno de la educación. No obstante, este método requiere de la utilización del supuesto de que los salarios se igualen a las productividades marginales y que las diferentes categorías de trabajo, clasificadas por años de escolaridad, se constituyan en sustitutos en la producción. Adicionalmente, el método supone perfecta movilidad de la mano de obra y que existe competencia perfecta en los mercados de trabajo.

El cálculo de la Tasa Interna de Retorno de la educación para los individuos perceptores de ingresos se constituye en una manera de medir los deter minantes del salario y sus efectos en la decisión de educarse y de acumular capital humano. De 
esta forma, si se considera una perspectiva de mediano y largo plazo sobre el crecimiento económico, es crucial la contribución que puede ejercer la variable educación; de ahí la necesidad de promover el mejoramiento de la for mación del capital humano para potenciar la capacidad productiva y la prosperidad del país.

Los modelos desarrollados en tal dirección intentan definir los deter minantes de los salarios y terminan por estimar la incidencia sobre los salarios de variables ligadas a la educación y la acumulación de aprendizaje, como son los años de escolaridad y la experiencia potencial. En el trabajo pionero de Human Mincer (1974) se establece la metodología del cálculo de la Tasa de Retorno de la educación mediante la estimación de funciones de ingreso laboral. Mincer encuentra evidencia empírica de la existencia de una relación de causalidad positiva, que va de educación a incrementos o mejoras en los ingresos laborales, lo cual es consistente con la tradicional Teoría del Capital Humano.

La educación es un bien de inversión y, como tal, constituye una contribución relevante al desarrollo económico. Toda inversión supone afrontar costos para obtener los beneficios esperados. La relación existente entre ambos puede ser analizada desde unadobleóptica: i) la privada (análisis financiero), que compara los costos y beneficios directos, valorados a precios de mercado, para escoger la alternativa que maximice el volumen y la tasa de ganancia para el dueño de los recursos que se asignan al proyecto; y ii) la social (análisiseconómico o socioeconómico), en la que se consideran también costos y beneficios indirectos a precios de eficiencia, buscando maximizar el impacto (rentabilidad) de la inversión sobre la sociedad en su conjunto.
Tanto en la evaluación privada como en la social se parte de un principio muy simple. Se comparan los beneficios (B) con los costos (C) del proyecto, y si los beneficios son mayores que los costos, se tiene una primera aproximación de que el proyecto debería, en principio, ser aprobado. Si B es mayor que C existe, en consecuencia, un excedente. Este debe ser mayor que el costo de oportunidad del capital utilizado en el proyecto. Para la evaluación financiera, el referente general de dicho costo de oportunidad está constituido por la tasa de interés existente en el mercado, denominado también tasa libre de riesgo.

Desde la perspectiva de la evaluación social, se considera el costo de oportunidad del capital a largo plazo, lo que se denomina tasa de descuento; ella es semejante a la tasa de interés, pero considerada desde la perspectiva de la sociedad en su conjunto. Es el costo del uso alternativo del capital en otro tipo de proyectos. Para el inversionista privado, los únicos costos y beneficios relevantes son los que se traducen, respectivamente, en egresos e ingresos; a estos se les denomina costos y beneficios directos. Pero el proyecto puede producir efectos que no se traducen en ingresos ni egresos; es decir, costosy beneficiosindirectos (o externalidades).

La polución producida por fábricas contaminantes es un costo indirecto, la disminución del tiempo de traslado de la población por la incorporación de un sistema eficiente de transporte es un beneficio indirecto. Ninguno de los dos entra en la contabilidad de la empresa, porque impacta a actores externos de la misma. Por lo tanto, no forman parte de su análisis; pero sí deben considerarse desde la perspectiva de la sociedad global. Por ello, la evaluación social considera costos y beneficios tanto directos como indirectos. 
La comparación entre costos y beneficios permite calcular la Tasa de Retorno de la Inversión, lo que constituye una guía para la asignación de recursos tanto en el ámbito privado como en el de la sociedad global, y así, sobre esa base, ella puede establecer prioridades inter $e$ intrasectoriales que se traducen en las políticas públicas. En materia de educación, la inversión puede resultar en varios tipos de beneficios que, si bien se encuentran interrelacionados entre sí, resultan analíticamente distinguibles: el aumento de conocimientos y destrezas que incrementan la productividad del trabajo, el crecimiento del producto nacional (beneficio para el país) y del ingreso (beneficio para el individuo), la disminución del tiempo que se requiere para el acceso al mercado laboral, lo que beneficia tanto al individuo como a la sociedad. Se puede estimar la contribución que la educación realiza a la productividad comparando las diferencias a lo largo del tiempo entre los ingresos percibidos por individuos que tienen diferentes capitales educativos. Aquí cabe resaltar lo siguiente: en los países donde la educación se encuentra fuertemente subsidiada, los costos sociales son mayores que los privados en una proporción que es función de la magnitud de las transferencias realizadas. Por lo tanto, la tasa de rentabilidad privada será, en esa misma medida, mayor que la social.

\section{EDUCACIÓN Y REMUNERACIÓN BASADA EN UN MODELO MICROECONÓMICO}

Si una persona tiene planeado seguir una carrera universitaria, sería necesario que evalué la inversión que va a realizar. Los costos en que incurre no solo serán explícitos, sino también implícitos, ya que tendrá que asumir un costo de oportunidad. Cuando se emprende una carrera universitaria que conduzca a un título profesional, se dejan delado variasopciones, justamente una de esas opciones son los ingresos que hubiera obtenido si solo se hubiera dedicado a trabajar. En todo caso, también podría ser lo que deja de ganar si es que su capital invertido en educación hubiera sido colocado a una tasa compuesta libre de riesgo en el Sistema Financiero.

El gráfico que se muestra a continuación ilustra claramente lo que sucede en casos típicos, cuando se inicia el programa de capacitación en educación superior y se asume el costo de oportunidad.

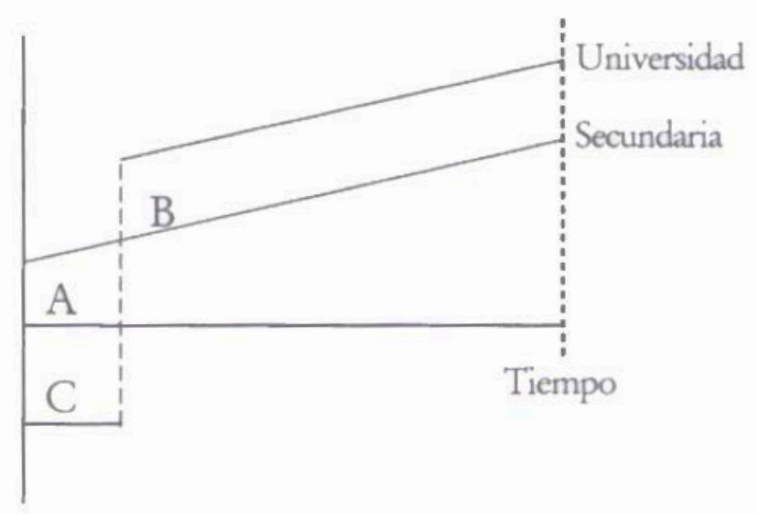

Gráfico N. ${ }^{\circ}$. Ingresos por nivel educativo logrado. 
$\mathrm{El}$ área $(\mathrm{C}+\mathrm{A})$ representa los ingresos que obtiene la persona cuando se está educando, el cual es negativo, que bien representaría la inversión que realiza para optimizar su valor como profesional. Después de lograr el título universitario correspondiente a la carrera elegida y dependiendo por cierto de su capacidad competitiva en el mercado laboral, el área " $B$ " representaría el ingreso adicional que obtiene como producto del valor agregado que le genera el programa de capacitación en educación superior. Es decir, sus ingresos son mayores cuando obtiene el título universitario en comparación cuando solo se queda con la educación básica, pero este último es lo que asume a manera de costo implícito cuando entra a la universidad.

Sin duda, la capacitación es importante para mejorar la capacidad de competitividad en el mercado laboral, el cual se debe de traducir en una mejora del ingreso, tal como se explica en el Gráfico N. ${ }^{\circ}$ 2, donde se correlacionala variable tiempoen educación y la variable ingreso o salario.

$$
\mathrm{W}=\mathrm{F}(\mathrm{g})
$$

Donde la dy / dtc = Ingreso marginal cuando se adiciona una hora en capacitación, también se le denomina retorno marginal de la capacitación

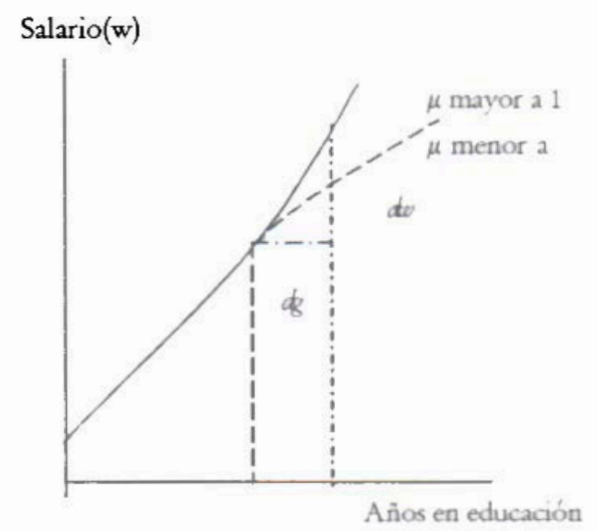

Grafico N. ${ }^{\circ}$ 2. Salario y capacitación.
Para que el impacto de la educación en los niveles de ingreso sea positiva, la relación entre la variable dependiente $e$ independiente tiene que tener el mismo comportamiento, donde además el grado de correlación $(r)$ y el de determinación $\left(r^{2}\right)$ deben presentarel mismo signo perocercano a la unidad, con lo cual quedará validado el modelo. La misma característica deberá presentar la pendiente de la línea de regresión.

Si se tienen datos estadísticos sobre estas dos variables, se podría estimar la ecuación de regresión. En el primer caso tendría que ser una lineal simple, con un â positivo.

La ecuación sería:

$\mathrm{W}=\mathrm{a}+\beta(\mathrm{w})+£$

$£=$ variable aleatoria

Porotrolado, en el $(\mathrm{dw} / \mathrm{dg})=\mu$, llamado también tasa de retorno de la capacitación, para que se justifique la inversión en estos programas a través del tiempo es importante que $\mu$ sea mayor a 1 ; en el caso que esta tasa sea menor a 1 , la inversión en capacitación no tendrá un retorno mayor en tér minos de unidades remunerativas, lo cual sería algo atípico. Si $\mu$ es menor a 1 , se presenta un problema grave, ya que la tasa de retorno de la capacitación se hace negativa. ¿Suceden estos casos en la vida real? Seguro que sí. Para darnos cuenta solo basta dar un vistazo a lo que ocurre con las remuneraciones de los empleados del Estado, específicamente aquéllos que están involucrados en la carrera docente, ya sea a nivel básico como superior (veáse Gráfico N. ${ }^{\circ}$ ). 


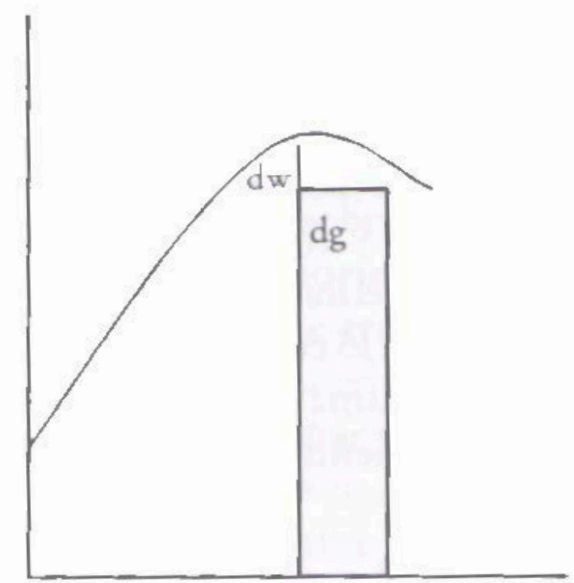

Gráfico N.o 3. Impacto negativo de la educación en el de la remuneración.

Hasta este punto se ha hecho un análisis recurriendo al instrumental macroeconómico.

\section{LA DECISIÓN DE INVERTIR EN EDUCACIÓN BASADA EN EL MODELO DEL VAN Y DEL TIR}

Para aplicar este método es preciso identificar las siguientes variables

$$
V A N=f\left(Y_{s / b}, E_{s / b}, I_{c}, r, N\right)
$$

$\mathrm{Y}_{\mathrm{s} / \mathrm{b}}=$ Ingreso obtenido con educación superior y básica

$\mathrm{E}_{\mathrm{s} / \mathrm{b}}=$ Costos explícitos en educación superior y básica

$i_{i=}$ inversión en educación superior

$r=$ tasa de descuentoo costodeoportunidad
$\mathrm{N}=$ Periodo de tiempo que se toma para evaluar ambas alternativas. Periodo que duran los estudios universitarios.

Como bien se sabe, no se puede comparar directamente cantidades en distintos momentos en el tiempo. El mismo monto monetariopercibido en el futuro vale menos que el percibido hoy. La tasa de descuento $r$ es la medida de cuánto menos vale el futuro en relación al presente. (Para recibir $\mathbf{X}$ unidades monetarias en el futuro, se está dispuesto a desprenderse hoy de una suma menor que $\boldsymbol{X}$; cuánto menos, eso lo indica la tasa de descuento. ¿Cuánto se debe recibir en el futuro para que se acepte desprenderse de un peso hoy?) El Valor Actual Neto traduce una suma que se recibe en el futuro a su equivalente monetario en el momento presente. La división de una cantidad que se percibe en el futuro por $(1+$ r) es su valor actual (o valor descontado).

$$
\mathrm{P}=\mathrm{F} /(1+\mathrm{r})^{\mathrm{t}}
$$

De esta relación matemática, se desprende la fórmula del VAN para evaluar proyectos:

$$
\mathrm{VAN}=\mathrm{I}+\sum_{\mathrm{t}=1(\mathrm{\Sigma}(\mathrm{Y} . \mathrm{E} .)}^{\mathrm{n}}
$$

Para dar operatividad al modelo se plantean las siguientes situaciones:

\begin{tabular}{|cc|}
\hline \multicolumn{1}{|c|}{ Situación 1 } & \multicolumn{1}{c|}{ Situación 2} \\
\hline \multicolumn{1}{c}{ Con educación universitaria } & \multicolumn{1}{c|}{ Con educación básica } \\
\hline $\begin{array}{l}\text { 1. Inversion en educación universitaria: } \\
\text { S/. } 30000 \text { (por toda la carrera) }\end{array}$ & $\begin{array}{l}\text { 1. Inversión en educación universitaria: } \\
\text { (S/.15 000) }\end{array}$ \\
\hline L. Ingresos estımados: 3000 mensual & 2. Ingreso mensual: 1200 \\
\hline 3. Lostos directos e indirectos: $50 \%$ & 3. Costos directos e indirectos: $30 \%$ \\
\hline 4. Periodo de evaluación: 5 & 4. Periodo de evaluación: 5 \\
\hline
\end{tabular}


Después de terminar los estudios universitarios, ya con el grado correspondiente, es de esperarse que el ingreso promedio que se obtiene en el mercado laboral puede llegar a S/. 3000, que es producto de una inversión que llega a los $\mathrm{S} / .30000$. Como se puede apreciar en el cuadro, el desembolso por este concepto es negativo en $S / .15000$, que en realidad es lo que se hubiera obtenido si no se hubiera seguido estudios superiores, es decir, lo que deja de ganar por estudiar cinco años en la universidad.

Para obtener el VAN, es necesario estructurarun flujo de caja dondese planteen las dos situaciones de esta manera se obtiene un flujo marginal, el cual es como sigue:
Aplicando la formula del VAN se tiene el siguiente resultado

$$
\begin{gathered}
\operatorname{VAN}=(45,000)+10,800 \frac{(1+0,10) \underline{5}-1}{(1+0,10)^{5} \mathrm{x}} \\
0.10
\end{gathered}
$$

$$
\mathrm{VAN}=-3690.46
$$

En este caso, estudiar una carrera universitaria, bajo los supuestos planteados, implicaría asumir una pérdida, es decir, la mejor opción alternativa le va a generar una ganancia de S/.-3690.46 que la decisión de estudiar una carrera universitaria. Para revertir esta pérdida lo que le queda al

\begin{tabular}{|c|c|c|c|c|c|c|}
\hline Periodos & 0 & 1 & 2 & 3 & 4 & 5 \\
\hline Inversión & 30,000 & & & & & \\
\hline Ingresos & & 36,000 & 36,000 & 36,000 & 36,000 & 36,000 \\
\hline Egresos & & 18,000 & 18,000 & 18,000 & 18,000 & 18,000 \\
\hline Beneficios $\left(B_{s}\right)$ & $-30,000$ & 18,000 & 18,000 & 18,000 & 18,000 & 18,000 \\
\hline
\end{tabular}
"inversionista" es ajustar su inversión en capacitación universitaria, es decir buscar

Flujo son educación superior $\left(\mathrm{B}_{\mathrm{s}}\right)$ (Nuevos soles)

Flujo con educación básica (secundaria) $\left(B_{\mathfrak{b}}\right)$ (Nuevos soles)

\begin{tabular}{l|c|c|c|c|c|c|}
\hline Periodos & 0 & 1 & 2 & 3 & 4 & \multicolumn{1}{c|}{5} \\
\hline Inversion & $(15,000)$ & & & & & \\
\hline Ingresos & & 14,400 & 14,400 & 14,400 & 14,400 & 14,400 \\
\hline Egresos & & 7,200 & 7,200 & 7,200 & 7,200 & 7,200 \\
\hline Beneficios $\left(\mathrm{B}_{\mathrm{b}}\right)$ & 15,000 & 7,200 & 7,200 & 7,200 & 7,200 & 7,200 \\
\hline
\end{tabular}

Beneficio marginal (Nuevos soles)

\begin{tabular}{l|c|c|c|c|c|c|}
\hline Periodos & 0 & 1 & 2 & 3 & 4 & 5 \\
\hline Con educación superior $\left(\mathrm{B}_{\mathrm{s}}\right)$ & $-30,000$ & 18,000 & 18,000 & 18,000 & 18,000 & 18,000 \\
\hline Con educación Básica $\left(\mathrm{B}_{\mathrm{b}}\right)$ & 15,000 & 7,200 & 7,200 & 7,200 & 7,200 & 7,200 \\
\hline $\left.\mathrm{B}_{\mathrm{s}}\right)-\left(\mathrm{B}_{\mathrm{b}}\right)$ & $-45,000$ & 10,800 & 10,800 & 10,800 & 10,800 & 10,800 \\
\hline
\end{tabular}


una universidad más cómoda, oen todo caso ajustar para arriba sus niveles de ingreso después de graduarse. Supongamos que toma la segunda opción, la cual estima aumentar en un $20 \%$, el flujo quedaría como sigue:

Beneficio marginal (Nuevos soles)

\begin{tabular}{|c|c|c|c|}
\hline Periodos & $\begin{array}{c}\text { Con } \\
\text { educación } \\
\left.\text { superior ( } \mathrm{B}_{\mathrm{s}}\right)\end{array}$ & $\left.\begin{array}{c}\text { Con } \\
\text { educación } \\
\text { básica }\end{array}\right)$ & $\begin{array}{c}\left(\mathrm{B}_{\mathrm{s}}\right) \\
\left(\mathrm{B}_{\mathrm{b}}\right)\end{array}$ \\
\hline 0 & -30000 & 15000 & -45000 \\
\hline 1 & 19800 & 7200 & 12600 \\
\hline 2 & 19800 & 7200 & 12600 \\
\hline 3 & 19800 & 7200 & 12600 \\
\hline 4 & 19800 & 7200 & 12600 \\
\hline 5 & 19800 & 7200 & 12600 \\
\hline
\end{tabular}

$\mathrm{VAN}=\mathrm{S} / 2,512,65$

TIR: $12.37 \%$

$\mathbf{r}=10 \%$ (Costo de oportunidad. Tasa libre de riesgo. Tasa de rendimento mínumo exigible)

\section{"El rendimiento de la decisión de estudiar una carrera universitaria es mayor que el rendimiento mínimo que se exige"}

En este caso, emprender una car rera universitaria le generaría al "inversionista" un beneficio de S/. 2512.65 por encima de la mejor opción alternativa. La TIR de la educación superior estaría por encima del costo de oportunidad, es decir, que la rentabilidad de la inversión, en términos porcentuales, supera al rendimiento mínimo que se exige por el emprendimiento que se realiza. Sin duda, si se presenta este perfil, la mejor opción es ir a la universidad. La representación gráfica de estos resultados se detalla a continuación:

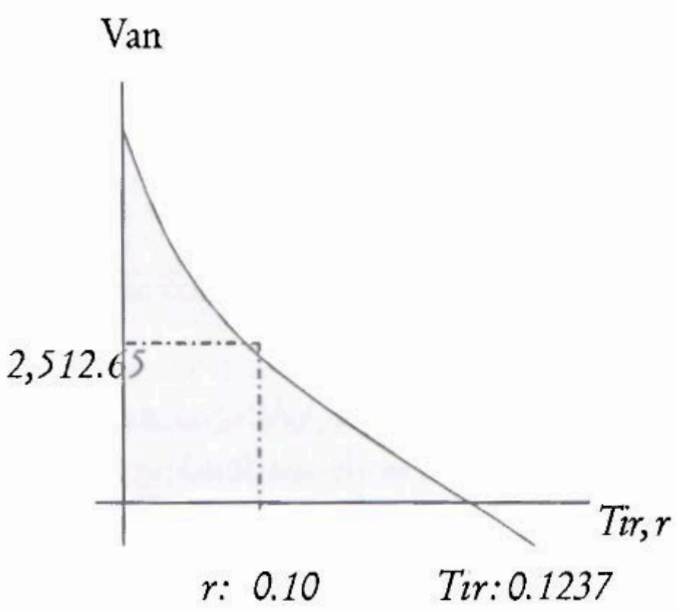

Gráfico N. ${ }^{\circ} 4$. Rentabilidad de la inversión de estudiar en la universidad.

\section{Propiedades:}

- Si la tasa de descuento o la tasa mínima de rendimiento exigible es más alta, menor será la rentabilidad de seguir estudios superiores o universitarios.

- Cuanto mayor sea la inversión en educación o lograrun título universitario, mayor tendrán que ser los flujos de ingresos para compensar dicha inversión o en todo caso el flujo de los beneficios netos.

- Si se presentara una mayor competencia en la carrera elegida, mayor será el castigo sobre los salarios profesionales, lo cual induce a una desmejora de la rentabilidad en educación, medida a través del VAN.

- Si lo que se deja de ganar por seguir estudios universitarios es mayor a la opción alternativa de estudiar, el impacto en la rentabilidad de este último será negativa. 
- Cuanto mayores sean los costos explícitos, después de terminar la carrera universitaria, y si no estuviera compensada con un mayor nivel de ingresos, la rentabilidad tenderá a caer. En todo caso se necesitará más tiempo para recuperar la inversión en educación.

¿Qué pasaría con la rentabilidad de estudiar, si se asume que existe una tasa de riesgo, que bien podría ser la probabilidad de no encontrar lo que se tenía planeado en términos laborales. La fór mula del VAN quedaría como sigue:

$$
\mathrm{VAN}=\mathrm{I}+\sum_{\mathrm{t}=1}^{\mathrm{n}} \frac{\left(\mathrm{Y}_{\mathrm{si}}-\mathrm{E}_{\mathrm{si}}\right)}{(1+\mathrm{r}+\bar{\beta})^{\mathrm{t}}}
$$

Tasa de riesgo
Cuanto mayor sea ß, o el riesgo, menor será la motivación por seguir una carrera universitaria, ya que la rentabilidad por esta opción tendería a caer. Esta situación es típica en mercados laborales deprimidos o con pocoespacioparaabsorbermanodeobra que egresa de las universidades. Otro factor que influenciaría en el valor de R, sería el tipo de universidad y la calidad de la educación, cuanto menos sea la exigencia académica el desprestigio de este centro de estudios aumentay con ello el nivel de riesgo, desmejorando o castigando la rentabilidad de invertir en educación. La demostración gráfica es como sigue:

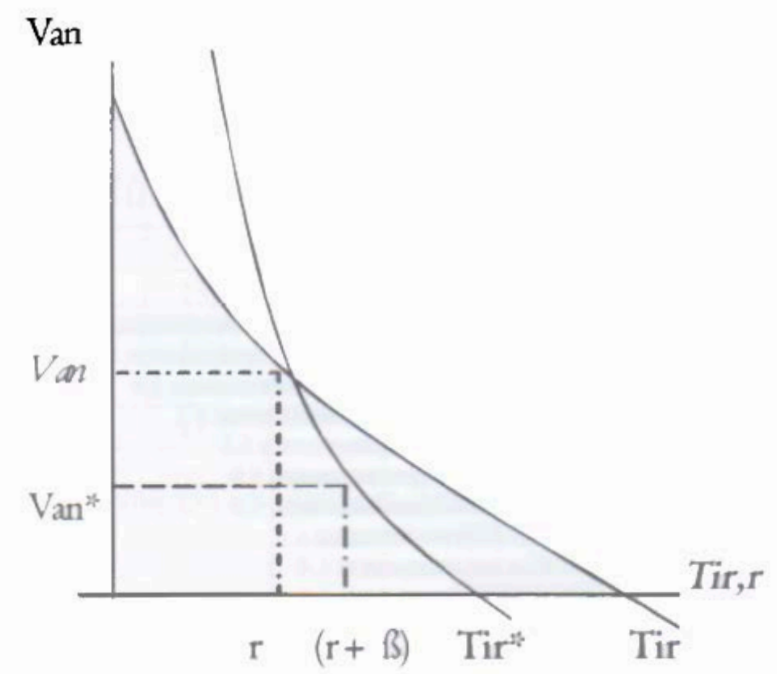

Van ${ }^{*}=$ rentabilidad con tasade riesgo
Van $=$ rentabilidad sin tasa de riesgo
Tir $=$ Tircon riesgo
Tir $=$ Tir sin riesgo

Gráfico N. ${ }^{\circ}$. Rentabilidad de la educación incluido el riesgo. 


\section{CONCLUSIONES}

1. La educación es prioridad indispensable para que un país logre su crecimiento y desarrollo económico, cuyo resultado se visualizará a largo plazo. El capital humano, correlacionado con el intelectual, es el componente básico para todo proceso de crecimiento con bienestar, para ello lo primordial es una educación con calidad, tema que debe de ocupar el primer lugar en las agendas de gobierno.

2. El retorno económico dela educación no solo debe evaluarse desde el punto de vista privado, sino también desde el punto de vista social, ya que delos efectos multiplicadores que ejerzase podrálograr una economía con mayores fundamentos para crecer en forma sostenida.
3. La evaluación de invertir en capacitación se puede realizar apoyándose en la teoría microeconómicay financiera, instrumento importante para tomar decisiones en este campo. El objetivo es obtener una rentabilidad superior por estudiar o capacitarse, que otra mejor opción alternativa. Pero este objetivo debe estar eslabonado con los objetivos del país.

4. Invertir en educación implica asumir un riesgo que castiga a la rentabilidad. El riesgo no solo está en el mercado laboral, sino también en el centro superior o universidad, donde se ofertaconocimientos. El desprestigio de una universidad, que está asociada a turbulencias políticas internas y debilidades académicas, alimenta el riesgo de quien quiere invertir en educación.

\section{ANEXO \\ INFORMACIÓN RELEVANTE SOBRE LA INVERSIÓN EN EDUCACIÓN E INVESTIGACIÓN}

América Latina (22 países): Evolución del gasto público en educación como proporción del PIB (porcentajes)

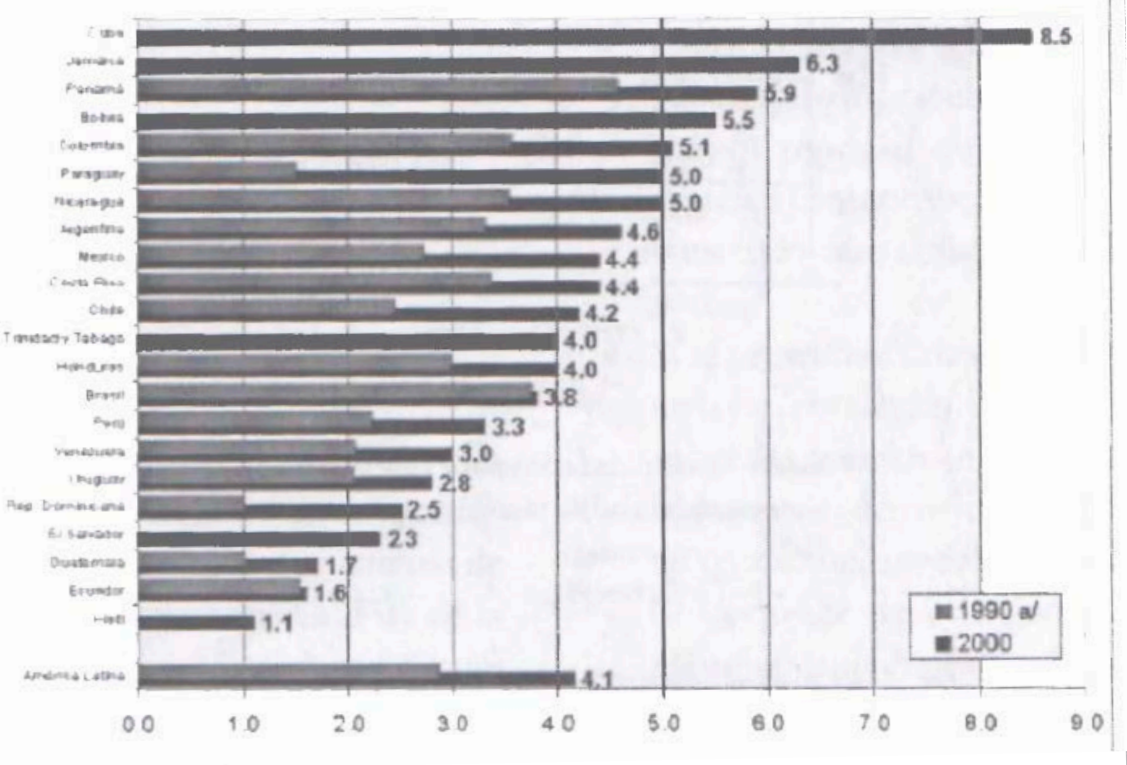

Fuente:ComisiónEconómicaparaAméricaLatir

dedatossobregastosocal. Corresponde al promedio simplede 16 países, excluidosBoliviay El Salvador. 
América Latina (18 países): Evolución del gasto público en educación por habitante (en dólares de 1997)

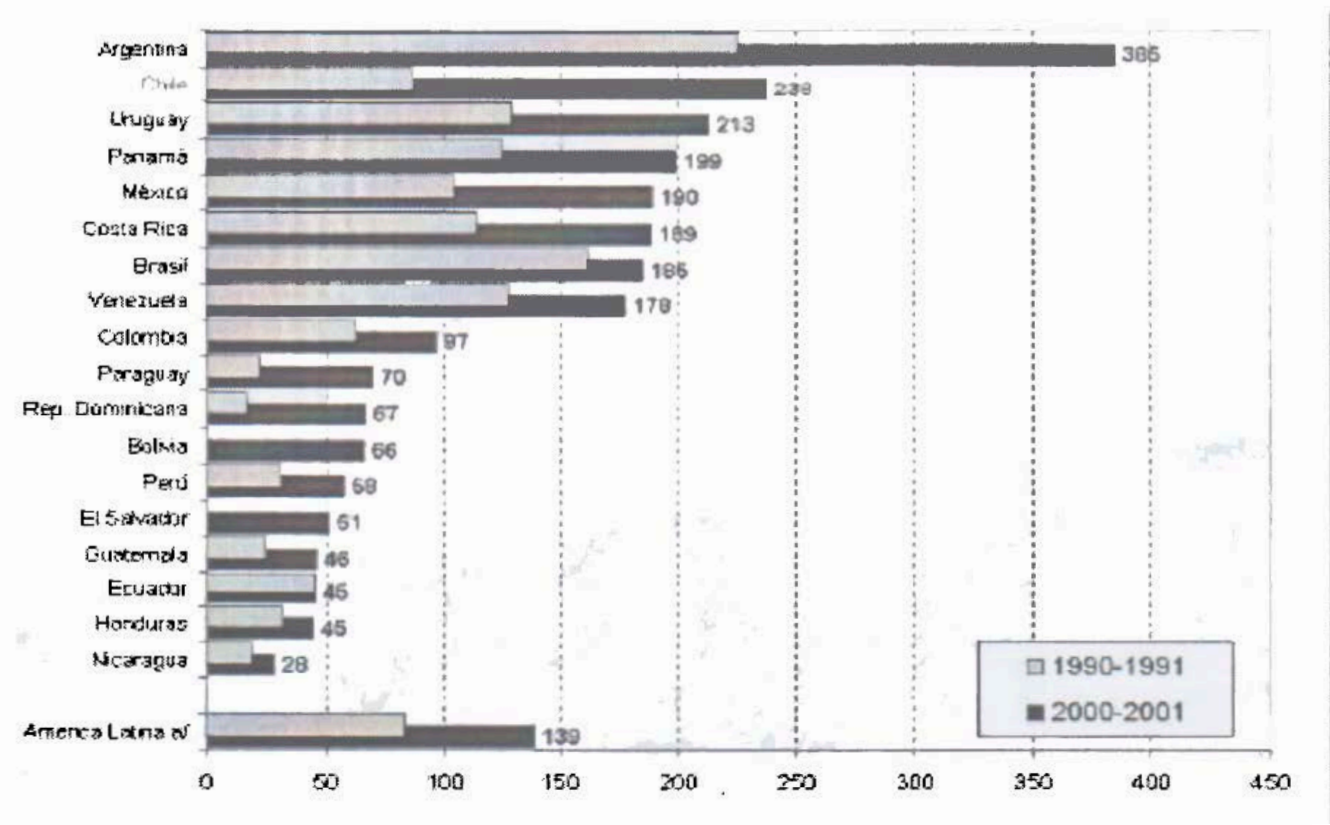

ruente: Compendro MundraldelaEducacion 2003. ComparaciónidelasEstadísticasdeEducaciónenelMundo, Montreal $\_$3, Instituto de Estadística de la UNESCO (UIS). Estimación basada en la evolución del gasto público social en arcación durantelos añosnoventa reportada porlaCE.

\section{Banco Mundial: Tasa de Rentabilidad de distintos sectores económicos}

\begin{tabular}{l|l|l|l|}
\hline \multicolumn{1}{|c|}{ Sectores } & $1974-1982$ & $1983-1992$ & $1974-1992$ \\
\hline -gncultura & 14,11 & & \\
\hline I-adustria & 15,0 & 12,0 & \\
\hline - Eraestructura & 18,0 & 16,0 & \\
\hline -.oucacion & & & 20,0 \\
\hline -odos los proyectos & 17,0 & 15,0 & \\
\hline
\end{tabular}

F_ente: Banco Mundial. Prioritiesand Strategiesfor Education. Washington, D.C.

\section{RIEFERENCIAS}

- Beyer H. "Educación y desigualdad de ingresos: Una nueva mirada". Estudios Públicos 77, Verano 2000.

- Beyer, H. "Distribución del Ingreso: Antecedentes para la discusión". En: Extudios Públicos. 65.

Helmuth Yesid Arias Gómez y Alvaro Hernando Chaves Castro. Cálculodela Tasa - nadeRetorno delaeducacionen Colombia, 2204.
4. Nassir Sapag Chain. Formulación y evaluación de proyectos. Editorial McGrawHill, Chile, 2000.

5. Pascale, Ricardo. Decasones financeras. 3. ${ }^{\mathrm{a}}$ ed., Ediciones Macchi, Argentina, 1998.

6. Pérez, Luis. Estadísticas básicas para Ciencias Sociales y Educación. Editorial San Marcos. Lima.

7. Las Ecuaciones de MINCER y las Tasas de Retorno a la educación de Chile. 